\title{
Parcours et détours en Écosse. Dark Earth de David
} Harrower

Danièle Berton-Charrière

\section{(2) OpenEdition}

1 Journals

Édition électronique

URL : http://journals.openedition.org/etudesecossaises/163

DOI : 10.4000/etudesecossaises. 163

ISSN : 1969-6337

Éditeur

UGA Éditions/Université Grenoble Alpes

Édition imprimée

Date de publication : 31 mars 2005

Pagination : 205-216

ISBN : 2-84310-061-5

ISSN : 1240-1439

\section{Référence électronique}

Danièle Berton-Charrière, "Parcours et détours en Écosse. Dark Earth de David Harrower », Études écossaises [En ligne], 10 | 2005, mis en ligne le 31 mars 2005, consulté le 07 septembre 2020. URL http://journals.openedition.org/etudesecossaises/163; DOI : https://doi.org/10.4000/ etudesecossaises.163 


\section{Parcours et détours en Écosse Dark Earth de David Harrower}

DANIÈLE BERTON-CHARRIÈRE Université StendhalGrenoble 3.
En 2003, pour le festival d'Édimbourg, le Traverse Theatre a demandé à quelques dramaturges d'écrire une pièce sur un thème commun: «Britishness». Parmi les auteurs retenus, David Harrower proposait une œuvre intitulée Dark Earth qui met en scène un détour campagnard, véritable parcours politique.

L'histoire de cette terre sombre met en scène deux jeunes citadins: Euan et Valerie. Un samedi, ils partent faire une promenade en voiture et ils tombent en panne dans une zone non urbaine particulièrement déserte. Pendant que l'un attend près du véhicule, l'autre se rend dans la ferme la plus proche pour chercher de l'aide. C'est ainsi que, séparément, ils rencontrent un des membres de la famille Cauldwell. Le jeune homme fait la connaissance de Ida qui ouvre la porte à laquelle il est venu frapper. La jeune femme, elle, est secourue par Petey qui la voit au bord du chemin sur lequel il passait en voiture.

Démarche ou hasard, c'est ainsi que des Écossais des villes rencontrent des Écossais des champs, et; c'est ainsi que naît l'histoire banale d'une coïncidence qui met en relation deux couples que rien, en dehors d'un incident mécanique, n'aurait amenés à se croiser. Les uns vivent en milieu urbain; ils sont pleins d'avenir et démarrent une carrière prometteuse; tout semble leur sourire même si leur couple connaît quelques soubresauts après deux ans et demi de vie commune. Les autres demeurent au cœur d'une nature dure mais pittoresque qui s'étend à perte de vue et que jouxtent çà et là les «vestiges » du mur d'Antonin.

La pièce donne à voir l'histoire triste d'une rencontre qui tourne à la confrontation. Sur scène, la mise en contact d'éléments détonants est expérimentale et David Harrower procède à sa mise en œuvre au cœur d'un laboratoire niché dans l'entre-deux des Basses et des Hautes Terres.

Ne pouvant plus se rendre à destination, les jeunes gens acceptent l'hospitalité de la famille Cauldwell dont la gen- 
tillesse devient de plus en plus envahissante, pesante, gênante et insupportable. Ida et Petey font preuve d'une chaleur humaine caricaturale carcan:

IDA : Cannae be that busy. Cup o tea for you there.

EUAN : No, I should be -

IDA : It's made now. Drink it. Aye, sit down.

$[\ldots]$

EUAN : Right, I should be off.

IDA: What for? See round here, dinnae finish a cup o tea someone's made you, causes offence. True. Seen it happen. Folk harbour it for years.

Parce que les uns sont détournés de leur itinéraire initial et parce que les autres sont économiquement contraints au départ, ils sont tous amenés à faire un bout de route ensemble, selon l'expression consacrée. Le mouvement dramatique général est intéressant car, comme les arabesques, il est fait de rencontres et de séparations, de convergences et de divergences.

Les deux Glaswégiens quittent leur ville réputée dynamique et, comme on le disait dans les années 1980, « on the move» pour une journée que Val voulait romantique. Journée de rupture avec la routine pour éviter une rupture affective subrepticement en marche. Elle a délibérément «oublié» les téléphones portables dans leur appartement. Le cordon ombilical ainsi coupé avec la vie citadine, ils vont à la campagne.

Leur parcours est interrompu par une panne d'embrayage. Est-il nécessaire de rappeler que cette pièce de mécanique permet de changer de vitesse et d'initier l'accélération ou la décélération opérées par d'autres? Le système rompu, la voiture n'avance plus, et Val et Euan se retrouvent «coincés» au milieu de nulle part où tout s'immobilise. Leur impatience, quasi juvénile, reflète l'inhabituelle staticité que l'incident leur impose. Ils perdent tous leurs repères et la carte qu'ils ont emportée ne leur semble pas d'un très grand secours. Ils essaient de l'interpréter et se disputent en vain. Les deux personnages découvrent qu'ils sont hélas sur une des pliures du précieux document. Dès le départ, leur position est donnée comme délicate et ambiguë, et leur situation semble inextricable, comme si le sort s'acharnait contre eux. Toutefois, la pièce n'est pas à l'image d'une tragédie classique et les jeunes gens ne manquent pas de ressources.

Leur seul point de référence devient alors le mur d'Antonin, ou ce qu'il en reste. De fait, n'étaient-ils pas partis de Glasgow 
pour le voir? Cette ligne de démarcation d'une soixantaine de kilomètres environ devient alors un repère sûr et fiable pour «se retrouver», «trouver son chemin» et «trouver le bon chemin». Vestige de la grandeur et de la ténacité de leurs ancêtres pictes, n'est-elle pas le symbole de la survie de l'Écosse et donc, pour Euan et Val, une emblématique raison d'espérer? C'est un indice maigre mais précieux pour re/trouver sa voie quand on est perdu dans ce labyrinthe ouvert. Le manque de culture patrimoniale et nationale des deux jeunes gens les contraint à la conjecture. Ce pays est le leur, mais l'univers tourbeux dans lequel ils viennent de s'échouer leur est inconnu et étranger, tout comme ils sont étrangers au monde rural que symbolise la famille de l'allégorique Petey Cauldwell'1. Tous les jeux de l'onomastique sont permis ici. Ils expliquent aussi l'attitude de chacun des personnages. Les Cauldwell se sentent privilégiés et métaphoriquement supérieurs aux jeunes gens: Val, diminutif de Valerie, rappelle la vallée sur laquelle se déversent toutes les eaux qui, selon Ida, deviendront stagnantes:

Petey: You won't be used tae that, will you, Val? Peace an quiet livin across there in that swamp.

VALERIE: Swamp? Is that what you think o us?

Petey: Naw. it's geography.

IDA: We're up high here - the water drains down either side. East an west. You get our slops.

Petey: The glacial shelf, Val, that's what it is. Nothin can be done about it.

Pourtant, le mur d'Antonin et la pliure de la carte sont à la fois des barrages («cauld») et des gués, des seuils. Selon les points de vue, ils sont obstacles ou protection. Ils poussent au franchissement, à l'intrusion. Mais, lieux de rupture, ils définissent aussi un entre-deux de rétention qui canalise la progression de l'autre. Topoï statiques, ils séparent deux mondes

1. «Petey» est homophone de «peaty»: l'homme et la tourbe peuvent être perçus comme des synecdoques traditionnelles de leur environnement. Métonymie du topos, le patronyme «cauld», signifiant «cold», «stiff» et "clayey", renvoie vers ses idiosyncrasies. Lembourbement qu'il connote retient l'homme prisonnier. et deux tranches de temps. Lavenir de Val et Euan mis entre parenthèses un moment, le drame de David Harrower donne ainsi à voir un parcours initiatique en milieu clos, un voyage au cœur de l'Histoire, véritable va-et-vient entre le révolu et l'actuel. Deux étapes y sont marquantes: l'invasion romaine et la tentative de restauration du pouvoir des Stuart. Ces deux épisodes se cristallisent autour du personnage de Christine Cauldwell, la fille, qui possède un livre sur la construction du mur et qui connaît en détail cette période: elle voue un véritable culte à Bonnie Prince Charlie. Source de savoir et de culture 
personnelle, sa passion pour la figure historique n'en est pas moins donnée comme ridicule, car elle est comparée à celle d'une jeune fan, déraisonnable et futile, pour son idole. Quant au livre, cadeau touchant et symbolique d'un père à sa fille, Euan essaiera de le lui voler, instinctivement, de façon compulsive, sans trop savoir pourquoi.

Les repères pris, la conjoncture évaluée, les deux jeunes décident d'agir pour éviter tout enlisement dans une situation inconfortable et désagréable. Le jeune homme quitte la voiture et son amie et il se met à marcher en direction d'une hypothétique habitation, source de vie et de secours. Il se déplace vers les autochtones. Sa démarche est forcée. Il n'a aucune envie de rencontrer l'habitant et de se faire héberger chez lui. Il veut juste contacter un garagiste ou la RAC, cordons ombilicaux de substitution. De son côté, son amie n'attend pas très longtemps. Petey Cauldwell lui propose de la ramener vers la ferme. Un fois arrivés là, les faux départs et la fuite vont alterner avec l'immobilité et l'immobilisme du locus.

La structure de la pièce offre un rythme parfois syncopé, parfois très lent. La confrontation de deux modes de vie et de pensée en est le motif. Chez les Cauldwell, le couple de Glasgow n'est apparemment pas en danger : il est à l'abri des intempéries et de la nuit. Mais le garagiste se fait attendre. Les voilà maintenant bloqués dans la ferme, où petit à petit, ils vont se sentir pris en otages : prisonniers de la situation rendue compliquée par l'inefficacité des services de dépannage qui font des allers-retours inutiles sans pour autant réparer la pièce défectueuse puisqu'ils n'arrivent pas à repérer le lieu de l'incident. Ils sont ainsi captifs de l'hospitalité de leurs hôtes et de la mauvaise humeur de leur fille Christine, particulièrement agressive. Tout au long de la pièce, les cinq personnages vont cheminer les uns vers les autres puis, un instant, les deux couples vont donner l'impression d'aller ensemble dans la même direction et enfin, comme des étrangers, ils vont se séparer sous les insultes et les propos haineux.

Le parcours effectué par les jeunes gens peut se calculer en miles et se suivre sur une carte, d'ouest en est, de Glasgow à Falkirk et ses environs parsemés de repères historiques et géographiques comme Callendar, Prestonpans et Creighill. Pourtant, le détour imposé par la panne les contraint à des parcours transversaux complexes et douloureux. Le couple va se perdre «des yeux et du cœur» l'espace de quelques heures, égarés 
qu'ils sont dans ce milieu étrange et graduellement hostile. Ils vont se chamailler et envisager un avenir commun, c'est-à-dire regarder ensemble dans la même direction, va leur paraître inconcevable. Puis l'épreuve va les souder dans un même combat contre l'adversité. Pourtant, tout au long de la pièce, la distance psychologique qui les sépare est rendue palpable. Tout à fait égocentrés, ils se réfugient dans des bulles de réconfort individuel.

Au Traverse Theatre, la scénographie bloquait la perspective spectatorielle par un mur oblique et haut contre lequel s'appuyait une véranda, bulle étroite et modulable, seul espace de vie et de jeu visible. Dans la pièce de David Harrower, l'isolement est étouffement. Il est contagieux. Tous les personnages ont besoin d'air et de liberté dans ce grand espace ouvert où paradoxalement ils se sentent confinés. La modernité et le progrès ont eu raison des fermiers. Leur hospitalité n'est pas gratuite et leur aide n'est pas désintéressée. Elles demandent un retour. Les fermiers ont vendu leurs terres à un voisin plus chanceux ou plus doué pour les affaires et leur survie dépend de leur mutation, c'est-à-dire de leur conversion: ils veulent se tourner vers le tourisme et faire de leur exploitation un Bed and Breakfast pour échapper à l'exil et aux HLM de Falkirk:

IDA: But what I'm sayin is it's no impossible. It's better than fossilisin isn't it, on some estate in Falkirk. Isn't it, Val? Better than fossilisin an then dying, trapped somewhere like that where we dinnae know a soul.

La réussite de leur entreprise passe par le coup de main qu'ils demandent aux deux citadins qui, comme dans les clichés, symbolisent le modernisme et le savoir. Valerie a quelques notions de comptabilité et Ida lui confie, sans honte, celle de la maison. Euan se moque des deux femmes parce que les connaissances de son amie dans le domaine se limitent à une année d'étude dans la spécialité qu'elle n'a pas approfondie depuis huit ans. De plus, stratégie mercantile oblige, les Cauldwell impliquent les citadins dans ce qui est plus un rêve qu'un projet parce qu'ils pensent que, de retour à Glasgow, ils peuvent leur faire de la publicité et tisser un réseau, véritable toile attrapetouristes. Leur notoriété se déplacerait de bouche à oreille comme le vent sur les bruyères mauves. Lironie de David Harrower affleure: qui ne pense que la communication est salvatrice de nos jours? Pourtant, la tentative est noble ou légitime, même si elle est désespérée. 
Ida fait peu à peu entrer Valerie dans son rêve empreint de fausse naïveté ou de fausse candeur. En réalité, les deux femmes ne sont pas aussi aveugles aux subtilités et aux méandres du monde économique qu'elles veulent bien le laisser paraître:

IDA : It's futures on that piece o paper there.

VALERIE : I know it is.

IDA : An you're goin tae need another column on the side there for the extras.

VALERIE : Extras? What extras? What're the extras?

IDA : Petey takin them tae the Wall like he took you - that's the extras. We could charge for that - no ? You'd have paid for that, wouldn't you ? Learnin all that knowledge.

Petey est sceptique; Euan et Christine franchement contre. Puis le doute se glisse par les interstices et les lézardes des désillusions et ils investissent graduellement les cerveaux et les cœurs. Lorsque l'évidence et la réalité refont surface, l'amertume et la haine s'emparent de tous les personnages et la rupture est définitivement consommée. Chacun retrouve son camp, son clan.

L’ironie dramatique souligne la désespérance. Le mur aurait pu/dû être aussi beau et solide que le mur d'Hadrien. Hélas, il se réduit à un fossé, symbole de force et de faiblesse, de fragilité, de fractures et de fêlures. Petey serait donc un guide touristique du presque vide et du presque néant. La nature organique et emblématique du mur a donné son titre à la pièce: Dark Earth. David Harrower prend la peine d'en donner la définition en incipit :

DARK EARTH buried soil found in archaeological excavation which often reflects prolonged periods of abandoned settlement; alternatively, deposits of silty soil reworked by earthworms to produce grassland.

L'expression est évidemment polysémique puisque c'est dans ce terreau que David Harrower trouve ses racines et celles de l'Écosse moderne qui tente de se démarquer et de transgresser les seuils de l'Histoire porteuse de tragédies et de clichés exotiques et folkloriques. La virée d'un samedi à la campagne décidée à la hâte par Val et Euan ${ }^{2}$ tourne court et se

2. Euan ne serait-il pas envisageable comme la synecdoque du demitour ou «U turn»? métamorphose en un retour aux sources, un voyage au cœur d'une histoire où le privé et le national se confondent et s'affrontent. Mais ces fondations/fondements patrimoniaux, aussi 
riches d'enseignement et de matière soient-ils, s'avèrent inutiles dans la société moderne: Christine n'a pu les exploiter dans le système scolaire:

CHRISTINE: Well, I'm no goin anywhere. School was torture enough. Why'd I want tae prolong the pain o that? They'd never give you a chance to learn what you really wanted tae. Sit an listen tae all kinds o subjects I couldnae give a toss about and then they couldnae understand me wanting to know everythin I could about what really interested me. Where I live. Round here. I know all about round here. All there is tae know. I'd tae learn most of it myself cause with them it was always, right, we need tae move on now but I never wanted tae. I know about the Romans an their empire an Agricola an Antonius an the Votadini and the Selgovae. I know about Charlie an the seven men o Moidart an the genius Lord George Murrau an old Jamie the Rover bidin hid time in Rome. I know more people from round here joined him than any o them fuckin Highlanders. You don't know any o that, do you? Or about dairy herds and set-aside and cereal prices. I know about where I live.

Pas un instant, elle ne croit que cela puisse intéresser qui que ce soit :

Christine: What's it like then? Tell me. Standin on a garrison station starin across a strange, unfamiliar wilderness, God knows who's lyin in wait over the horizon. (Pause) What did he tell you? We used tae swap sides just for the hell o it, choose a side o the wall tae stand on - tae get the argument goin. The Wall was a perimeter or a line o defence? What was he sayin?

VALERIE: That it was a perimeter.

CHRIsTINE: Cause who'd want north o here? All those mountains an bogs. Waste o time an manpower, eh? An who'd want tae go tae the bother o fighting them stinkin Caledonian hordes, eh? Naw, leave them tae scrap amongst themselves. Was that his line?

Le locus dramatique est marqué par cette ligne de terre que certains voient en creux et d'autres voient en plein. Équidistant entre Glasgow et Édimbourg, près du mur d'Antonin, il est perdu en pleine nature. La rencontre entre les deux groupes de personnages aboutit au huis clos. La véranda où se concentre l'action est un topos de l'entre-deux. Dans la pièce de Harrower, elle affiche une fonction paradoxale: symbole d'ouverture sur l'espace extérieur alentour vaste et rafraîchissant - vivifiant, - elle est la vitrine de l'enfermement et de l'isolement des Cauldwell et de la nation. Christine y est retenue prisonnière par l'amour de ses parents. Elle a 20 ans et pourtant sa 
maturité et son sens des responsabilités l'enchaînent à ses sources/racines. Ultime tentative de vivre sa propre vie de jeune femme moderne, elle décide de fuir avec Euan, mais en vain. Petey les rattrape et son discours affectueux et désespéré l'enlise et la reprend dans ses rets. Il l'embourbe, il l'englue à jamais dans ce «boggy land» qui n'est plus tout à fait le leur puisque leurs voisins, Duncan et Susannah Craig's, ont racheté leur propriété.

À l'image de celui qui a inspiré son prénom, Christine est sacrifiée sur l'autel d'une idéologie passéiste:

IDA : Christine? What's more important? We have tae survive. If it means sacrificin her - She should know by now what kind o people we are. We fight wi all we've got. It's best she's gone, it's best. She should've been out here long before now - but it was Petey. Petey held ontae her. She's her father's daughter. Could you see us leavin here? How would we? In daylight? No- the dead o night. We wouldnae be able tae look at each other, me an Petey. Our daughter, brought up here, leading us away? No. (Beat) Know what it is wi her? Know what it is wi Christine? She came too late. We had her too late.

Leur milieu est clos et le temps y a une place tout à fait particulière. L'événementiel y fait date et tous les raccourcis temporels y sont possibles par le jeu des mises en abyme de l'Histoire dans l'histoire.

Le dépanneur n'ayant pas trouvé la maison des Cauldwell, il est reparti: Val et Euan doivent donc accepter l'hospitalité de Ida et de Petey et ne repartir que le lendemain. Ce report, qui leur est imposé, porte un coup d'arrêt au départ et initie un retour sur le passé. Le problème immédiat résolu, tous les personnages se détendent. L'ambiance est joyeuse. On boit beaucoup de pur malt (du Bunnahabhain). On parle de souvenirs chaleureux et Ida demande à son mari et à sa fille de chanter leur chanson préférée comme dans le bon vieux temps des soirées entre voisins. Ils entonnent donc The Young Pretender d'Elvis Presley. Par le biais des associations d'idées et par le procédé du palimpseste, David Harrower entraîne les personnages dans une série de quiproquos fondés sur des jeux de superpositions.

3. Le signifiant «Craig» dénotant le rocher et ce qui est en hauteur, qui émerge, il peut être perçu comme leur blason. L'amoureux transi dont parle le rocker américain dans son tube international devient Bonnie Prince Charlie, héros romantique, tragique, et modèle charismatique pour Christine. Abusée par les plaisanteries de ses hôtes, Val croit un instant que l'on parle du petit ami de la jeune femme. Les discours jouent 
sur l'implicite et le non-dit, sur l'évident et le non-su, et le comique de situation s'en nourrit:

VALERIE: Is Charlie your boyfriend?

IDA: Her boyfriend - ! She wishes.

EuAN: Charles Edward Stuart, Val.

IDA: Bonnie Prince Charlie.

EuAN : The Young Pretender.

VALERIE: Oh, I didn't even realise! Oh, that's awful!

Christine: King James the Third tae you lot. The title he'd have used.

Petey: Pathetic Prince Charlie.

IDA: His army marched right past us here, Val, right outside, on their way tae Edinburgh.

CHristine: On their way tae take Edinburgh. On their way tae defeat jitterin Johnny Cope at Prestonpans.

IDA: Stayed the night just up the road there in Callendar House wi the Duke o Atholl.

En filigrane, l'auteur nous passe un tout autre message que rendent lisible des jeux de miroir et des échos. La réalité du héros auquel Christine et son clan s'identifient est pathétique: Bonnie Prince Charlie était alcoolique (Ida boit beaucoup trop et Valerie l'imite). Son errance après Culloden est emblématique du désir de fuite de Christine et d'une certaine catégorie d'Écossais velléitaires, à l'image de Petey et de Ida, qui se complaisent dans leur «statut» de victimes, éternellement coupables - victimes de l'Histoire et d'un certain déterminisme socioculturel. David Harrower accuse l'Écosse d'être fière de son statut de «loser's land»:

CHristine: Make it easier for you, Val, get you involved. You're from the west so you're one o the Damnonii. Your homeland's the Clyde Valley. A tribe that never made peace wi the Romans - we were the Votadini, we did, tradin an that. But always wanted tae know why you didnae. Me an my dad always admired you for that. What was it about them? Did you no trust them? What was it? $[\ldots]$

VALERIE: This area - the land around here - your dad said it was loser's land.

Short pause.

Christine: He said that?

VALERIE: There'd been victories, yeah, but most of it was losing, most of it was lost causes.

Ida, dont le prénom est phonétiquement modal, marque de son empreinte l'irréel du présent: que pourrait-elle encore 
4. Anachronismes et raccourcis temporels jouent sur l'enchaînement et l'association d'idées qui permettent de ramener à soi le général et l'historique: "VALERIE: I think the Romans just got homesick for Rome - for the sun and the ice - cream and the pavement cafés - I think that's why they left.»

5. Ida sits down./The book remains on the table between them./End. envisager à regret pour que se modifie la situation qui l'accable et qu'elle nie? Sa désappropriation, cette perte de biens, de patrimoine et d'identité, se lit aussi à la lumière des mesures de rétorsion et des «clearances» décidées en leurs temps par le pouvoir en place, après le passage de l'icône rebelle, symbole qui tient lieu et place de «gendre idéal» pour les Cauldwell. Les échos se répondent en un drame polyphonique.

Traverser l'histoire et se l'approprier leur permet tous les raccourcis, ciments de leçons morales faciles: Petey rappelle que les Highlanders n’ont jamais dénoncé Charles Stewart. Dans les Hautes-Terres, on ne trahit jamais sa famille et son clan. Christine doit se le tenir pour dit, elle qui tient à ce que toutes les décisions se prennent en famille et non individuellement. Ainsi, le libre arbitre est canalisé et le parcours de chacun semble bien balisé. Le passé délimite le présent. C'est probablement la raison pour laquelle, c'est hors de leur champ natal, c'est-à-dire à Falkirk, que Petey et Ida se sentiraient fossilisés et morts/mourants.

Mais pourquoi Charles Stuart leur serait-il si familier? Son passage fugace, près de leur maison, quelque deux siècles et demi auparavant, ne peut légitimer un tel attachement. Leur topos est aussi creux et terne que le mur qui le borde. Le rappel historique l'éclaire de son prestige, l'espace d'un instant, même s'il connote l'échec et le sang.

Euan n'est pas sous le charme de l'Histoire. Il se plaît à rappeler les failles de l'anti-héros. De fait, la vie de Bonnie Prince Charlie s'est terminée à Rome dans une oisiveté peu glorieuse. Le signifiant «Rome», symbole de grandeur et de décadence, initie d'autres détours mémoriels; Val se souvient de son séjour dans la capitale italienne, du soleil qui était chaud, de sa délicieuse immobilité et de ses cafés bondés et vivants ${ }^{4}$. Dans son désir hégémonique, Rome avait envoyé tant de ses soldats et de ses hommes sur les routes de la Gaule, de l'Angleterre et de la farouche Calédonie: la boucle est ainsi bouclée. Et c'est ainsi que David Harrower dit avoir travaillé. Ses incursions dans les livres d'histoire, travail préalable à l'écriture du drame, ont alimenté digressions et détours au gré de toponymes et de faits découverts ou repérés, mis en abyme çà et là dans la pièce. L'histoire du mur d'Antonin est inscrite dans le livre de Christine que Euan a tenté de dérober. Mais ni Euan ni David Harrower n'ont pu déposséder les Cauldwell du terreau culturel qui est le leur: la pièce se termine sur des didascalies ${ }^{5}$ indiquant que le 
rideau retombe sur le trio familial, assis dans la véranda. Seul le livre posé sur une table sépare Petey et Ida: mais les sépare-t-il vraiment? Ne les relie-t-il pas à jamais dans le fossé de leur passéisme, véritable tranchée terreuse nourrie du sang de leurs ancêtres? Leur départ avorté dans la voiture de Euan, «empruntée» un instant, semble l'indiquer. Tous les chemins les ramènent au point de départ. Ils tournent en rond:

EUAN : What the hell were you thinking of? That's my car. CHRistine: I wanted a go in it. Feel it for myself. I wanted tae take my parents out for a spin.

Lors de la rencontre auteurs/spectateurs organisée au Traverse Theatre en août 2003, David Harrower a déclaré ouvertement ne pas vouloir être un porte-parole, un dramaturge ostentatoirement militant qui défendrait la cause et l'écriture écossaises ou une toute autre cause politique d'ailleurs. Cette image qui lui colle à la peau, il la nie et dit l'abhorrer. Pour le prouver, il explique longuement à qui veut bien l'entendre qu'il monte ses pièces autour de lieux et de personnages et non autour d'un thème ou d'une histoire. Il revendique une écriture dramatique plus technique que politique. Il la veut centrée sur ce qu'il appelle «counterpointing». Toute chose est contrebalancée par son contraire ou par une autre qui est différente. Par exemple, l'hospitalité écossaise est rééquilibrée par le souci économique d'une société en pleine paupérisation ${ }^{6}$. Ou encore, ce qu'il définit de façon ironique comme «national mourning", c'est-à-dire la gloire et l'honneur dans la défaite, idiosyncrasies écossaises séculairement héréditaires, devenues la force et la survie de l'Écosse avec «Culloden» comme moteur qui génère le pouvoir et l'identité d'une nation!

Comme Spike Lee le fait pour les Noirs d'Amérique, David

6. Désespoir et pauvreté transforment l'hospitalité en traquenard: CHRISTINE: Freeloader! Thief! You're due us! Where's our money? EUAN: Money?

PeTEY: Aye, where is it? EUAN: We don't owe you anything.

CHRISTINE: One night's bed and breakfast. EUAN: It was hospitality last night, I seem to remember.
Harrower se délecte à démontrer que les Écossais sont multiples et différents. Au travers des personnages, il se plaît à opposer ceux de l'est trop pauvres à ceux de l'ouest plus riches, ceux des villes modernes et ceux des campagnes traditionnelles, ceux des Basses Terres et ceux des Hautes Terres, comme s'ils n'étaient pas tous dépositaires d'un même passé atavique. Ce n'est pas sans ironie que, l'espace d'un instant, David Harrower transforme Petey en porte-parole des fermiers de l'est qui blâment les ouvriers de l'ouest d'être à la source de leurs plus grands maux. Corporatiste et populiste à souhait, le raisonnement est absurde et simpliste, et Euan en reste pantois. Petey 
7. Petey: Aye. Cause an energy shortage meant that cattle-feed factorie werenae able tae heat their furnaces hot enough so's tae kill off all the bacteria. Listen, dinnae laugh. It's known. Healthy cattle were fed wi animal remains full o bacteria. That was the start o the BSE. Cows fallin in the fields. Beef banned. Nobody trustin us - naebody byin from us. That's how we're bein gotten rid o. affirme que les grèves des mineurs sont responsables de l'épizootie ravageuse. Il prétend qu'à cause d'elles, les usines d'équarrissage n'ont pas pu chauffer leurs produits à une température suffisante et qu'elles ont fourni aux paysans des farines animales contaminées par des bactéries. Ainsi nourries, leurs bêtes ont contracté l'ESB? .

Signes évidents d'un aveuglement et d'une étroitesse d'esprit que David Harrower condamne, les propos de Petey trouveraient assurément leur contrepoint chez les mineurs fustigés. La démonstration est patente: l'unité nationale se désagrège en une constellation de particules individuelles égocentrées. Le terreau dont parle le dramaturge résulte d'une lente décomposition organique naturelle. Il est emblématique et métonymique. Les terres dont Ida vante l'air si pur et frais grouillent de vieux démons à l'image du sol qu'aèrent les asticots. Et c'est dans ces terres sombres-là, qu'à la fin du drame, les Cauldwell s'enterrent vivants. 